\title{
LA INTERFERENCIA INTERSISTÉMICA COMO APROXIMACIÓN AL CANON
}

\author{
INTERSYSTEMIC INTERFERENCE \\ AS AN APPROACH TO THE CANON \\ Mario de la TORRE-ESPINOSA \\ Universidad de Granada \\ mariodelatorre@ugr.es
}

\begin{abstract}
Resumen: En el debate sobre el canon, la irrupción de las teorías sistémicas de la literatura propició el desarrollo de nuevos enfoques centrados en el análisis del proceso de canonización y desde una perspectiva diacrónica. Con la Teoría de los Polisistemas como marco teórico, proponemos la interferencia intersistémica, según Rakefet Sela-Sheffy, como indicio de canonización de un producto cultural en un polisistema determinado.
\end{abstract}

Palabras clave: Canon. Teoría de los Polisistemas. Teorías sistémicas. Interferencia sistémica. Teoría de la literatura.

Abstract: In the debate about canon, the emergence of systemic theories of literature has contributed to the development of new approaches centered in the analysis of canonization processes from a diachronic perspective. With the Theory of Polysystems as theoretical framework, we propose the intersystemic interference, as Rakefet Sela-Sheffy exposed, as a sign of the canonization of a cultural product in a polysystem.

Key Words: Canon. Polysystem Theory. Systemic Theories. Intersystemic interference. Literary Theory. 


\section{CANON EPISTÉMICO FRENTE A CANON VOCACIONAL}

Encontrar ejemplos de cánones objetivos que se distancien de forma clara de los cánones personalistas, elaborados a la manera de Harold Bloom (1994), es una tarea que en principio parece bastante compleja. La metodología del profesor norteamericano, la reivindicación de la estética por encima de cualquier consideración de otro tipo, implica que el juicio de valor emitido sea parcial e interesado, por mucho que se procure mantener dentro de unos límites académicamente equidistantes. Pero ciertamente una propuesta opuesta, estableciendo una relación entre la serie literaria o artística con el resto de las variables conformantes del sistema cultural, nos llevaría a cánones alternativos que solo pueden ser observados también como parciales, y por tanto insatisfactorios.

Ante esta disyuntiva, parece complicado encontrar una salida que concilie todos los posibles puntos de vista sobre este fenómeno. Por ello, como Enric Sullá (1998) apuntó, es reivindicable que en lugar de hablar de un canon en singular se hable de cánones en plural, de tal forma que todos los enfoques, por muy divergentes que parezcan, sean recogidos en un mismo listado o varios de ellos. Se trataría pues de contemplar todas las propuestas, independiente de las estrategias y metodologías seguidas. Con este modus operandi, tanto el proyecto de Bloom, como el de otras personas, independientemente de su orientación teórica - women studies, poscolonialismo, queer studies...-, conseguirían perfecto acomodo aquí a pesar de las perspectivas presentes.

Sin lugar a dudas este planteamiento conlleva indudables beneficios, ya que la no discriminación de ningún posicionamiento ideológico a la hora de hablar del canon parece a priori una decisión salomónica. Pero esta solución puede llegar a ser contraproducente si es llevada al extremo. Con el progresivo desarrollo de los Estudios Culturales y sus propuestas de canon, unido a las cada vez más numerosas voces que van planteando sus propios inventarios de obras, se corre el riesgo de que al final se elaboren listados de difícil aprehensión por cualquier sistema cultural. A pesar de que esta propuesta puede ser considerada como la más satisfactoria debido a su exhaustividad, el elevado corpus de obras que resultaría, así como la variedad de claves interpretativas puestas en funcionamiento, convertiría 
esta especie de mega-canon, múltiple e integrador aunque en demasía inflado, en un proyecto fútil a efectos prácticos.

La solución a esta discusión podría residir en la promoción de nuevos enfoques que intenten demostrar si realmente una obra artística se ha canonizado en el sistema o no, en lugar de determinar solo qué obras forman parte del canon, entendido este en su concepción tradicional como el listado de creaciones que debe ser conservado y transmitido posteriormente a través de las instituciones. Solo así se logrará un conocimiento ecuánime del funcionamiento del sistema. Siguiendo la terminología de Walter Mignolo (1991), se trataría de huir de los cánones vocacionales para intentar establecer un canon epistémico, verificado de acuerdo a criterios más objetivos $\mathrm{y}$, si se quiere, de un modo más científico. Y a este respecto las teorías sistémicas (Tötösy de Zepetnek, 1992), con la Teoría de los Polisistemas a la cabeza, se muestran totalmente competentes ${ }^{1}$.

El canon debería estar compuesto por obras que demuestren su condición de canonizada a través de una injerencia efectiva en el sistema cultural. Estaríamos así acercándonos a la idea de canonicidad dinámica enunciada por Itamar Even-Zohar, "a certain literary model that manages to establish itself as a productive principle in the system through the latter's repertoire" (1990a: 19), dirigiendo para ello la creación de otras obras en una sociedad determinada. Se enfrentaría así a la idea de canonicidad estática, aquella en la que "a certain text is accepted as a finalized product and inserted into a set of sanctified texts [that] literature (culture) wants to preserve" (1990a: 19), pero sin orientar la práctica artística en el sistema en el cual se inserta. En este último caso su impronta actúa esencialmente en el nivel de la institución académica o de otro tipo, sin llegar a afectar directamente a los productores culturales, al menos en ese determinado momento $^{2}$.

Además del análisis de las injerencias sobre la sociedad producidas por una obra o un modelo - normalmente introducido este mediante la

\footnotetext{
${ }^{1}$ Esta sería la idea compartida, entre otros, por José María Pozuelo Yvancos (1995, 2000, 2006), quien apuesta también por la Semiótica de la Cultura de Lotman por compartir con la Teoría de los Polisistemas algunos de sus principales presupuestos teóricos en cuanto al canon.

${ }^{2}$ La diferenciación que presentamos entre dos tipos de cánones sería también la enunciada por Rakefet Sela-Sheffy cuando distingue, para designar a estas dos formas de comportamiento, entre guide for cultural production y high status (2002: 147).
} 
presencia de normas coincidentes en varias obras - tras su canonización, hay otro aspecto esencial que es de especial relevancia por las implicaciones teóricas que acarrea: el concepto de durabilidad aplicado a la canonización. En primer lugar hay que señalar que el estudio de la construcción del canon debe ser afrontado desde una concepción diacrónica, ya que solo así es posible abordar en toda su complejidad la naturaleza de este fenómeno. Fijar este acontecimiento a un momento concreto solo nos permitiría abordar un análisis historiográfico parcial, sin llegar a entender el proceso completo de desplazamiento del producto cultural desde una posición marginal a otra más central en el sistema.

Relacionando esta variable temporal con los dos tipos de cánones mencionados podremos contemplar ciertas implicaciones nada triviales. Así, cuando una obra se convierte en un modelo para la creación de otros productos culturales — canonización dinámica - estamos asistiendo a un canon en presente que ejerce su impronta sobre el sistema cultural de forma determinante. En cambio, cuando se convierte en un elemento estático, se trataría de un canon para el futuro. En este último caso, su poder dentro del sistema cultural vendrá determinado tanto por el peso de la institución, que garantiza su posición central, como por su potencialidad para guiar la creación de futuras generaciones: lo que es canónico pero no actual puede cambiar su posición dentro del sistema con el tiempo y pasar a convertirse en un elemento rector en la creación de otras obras artísticas. Es decir, esta naturaleza estática es temporal y fijada por diferentes actores, pudiendo mutar a un carácter dinámico con el paso del tiempo y con los cambios acaecidos en el sistema. Este canon estático estaría compuesto pues por modelos presentes en el repertorio cuyo valor estético es reconocido por la institución, pero cuyo valor práctico es bastante reducido, ya que no afecta ni al mercado ni a los productores.

Esto implica que en la dinámica capitalista mundial el mercado imponga otros modelos de mayor rentabilidad económica, corriendo estos elementos del canon estático el peligro de quedar marginados por otros modelos más rentables - piénsese en el caso del habitual desinterés por la literatura grecolatina frente a la continua demanda popular de best sellers, a pesar de su consideración de literatura de menor calidad literaria-. A este respecto la institución, tal y como la describió Frank Kermode (1998), tiene una responsabilidad plena. Su ejercicio de poder, no solo a la hora de perpetuar obras en el repertorio sino también sus lecturas, es determinante 
en la conservación de ese high status descrito por Sela-Sheffy (2002). Este fenómeno de canonización se muestra por tanto, desde un punto de vista epistemológico, mucho más difícil de discernir, puesto que nos obliga a jugar habitualmente en el ámbito de las conjeturas. Su valor viene designado en la mayoría de los casos por juicios de valor aposteriorísticos prescritos por un horizonte de expectativas o Horizonterwartung previo (Jauss, 2013) que ejerce su autoridad a través de un ejercicio de selección, dificultando de este modo un conocimiento objetivo del canon al excluir elementos instalados normalmente en la periferia del sistema. El canon se muestra así manipulable por diferentes condicionantes, por muy bienintencionados que puedan parecer los criterios puestos en acción durante todo el proceso.

Una lectura de la idea de sistema cultural y sus mecanismos de acción desde la Teoría de los Polisistemas podría darnos la clave para realizar una aproximación verdaderamente fiel a la realidad, en cuanto que lo que se tiene en cuenta son las dinámicas implicadas en el sistema cultural, definido este como un ente dinámico sujeto a diferentes tensiones ejercidas tanto por el centro como por la periferia del sistema. Desde este paradigma teórico, la observancia de estos mecanismos nos puede dar la clave para identificar si una obra determinada ha sido canonizada o no, y cuál es su verdadera impronta en una sociedad dada. Al fin y al cabo esta es la función del canon: influir tanto en la creación como en la recepción en un marco cultural determinado, actuando como garante de unas ciertas claves culturales identitarias.

A este respecto los factores del sistema expuestos por Even-Zohar (1997) son de gran utilidad, ya que al contemplar las diferentes variables involucradas en cualquier fenómeno sociocultural - a partir del esquema de la comunicación de Jakobson (1960) — se abre las puertas a un abordaje holístico, que trasluce también una intencionalidad cientifista. Teniendo en cuenta que las relaciones entre estos factores son los que realmente constituyen el polisistema (la cultura), el análisis del rol del productor, del consumidor, del mercado, del producto, de la institución y del repertorio nos lleva a una lectura funcional y diacrónica del fenómeno artístico. Casos evidentes como la acción de la editorial Seix Barral sobre el boom latinoamericano de los sesenta y setenta vendría a apoyar esta visión. Y más si tenemos en cuenta las peripecias de la novela Cien años de soledad - rechazada por Seix Barral y publicada finalmente por la editorial 
Sudamericana - y de su autor, Gabriel García Márquez, hasta que se le concedió el premio Nobel. Viene a demostrar cuán interrelacionados están el factor productor (escritor) y el producto (novela) con el mercado (editorial), o bien con la institución (premios) que enalteció su realismo mágico (repertorio) y convirtió a su obra, junto a la acción del resto de factores, tanto en un auténtico best-seller (consumidor) como en un referente máximo (canon) en la literatura hispanoamericana y, por extensión, universal.

\section{LA INTERFERENCIA COMO MANIFESTACIÓN DE LA CANONIZACIÓN}

Además de los análisis que se puedan llevar a cabo a partir de los factores expuestos más arriba, tomando datos de diferente índole que alejan a estos estudios de los tradicionales en el ámbito literario, hay fórmulas que permiten identificar si un elemento ha sido canonizado o no. Entre estos hay que destacar aquí el de la interferencia por su operatividad y por la coherencia que aporta desde un punto de vista metodológico. Mediante este concepto Sela-Sheffy (2003) identifica aquellos fenómenos en los que, desde un posicionamiento intersistémico, un producto cultural o modelo ejerce sobre un sistema ajeno una influencia determinante en la ejecución de nuevas obras. Desde nuestra perspectiva, vamos a proponer este tipo de fenómeno como muestra del grado máximo de canonización.

A veces este acontecimiento tiene lugar de espaldas a la institución, ya que la canonización puede llegar antes de que esta advierta el cambio producido en el sistema cultural. Esto se debe a que a menudo estos desplazamientos se desarrollan en sectores de la sociedad al margen de los cauces oficiales, aún tratándose de actividades de naturaleza mainstream. Evidentemente no todas las prácticas culturales que se canonizan tienen que pertenecer a la high culture, es decir, no tiene por qué existir una correspondencia plena entre lo que la institución promulga y lo que realmente acontece en el resto del sistema cultural. Esta situación provoca una cierta desconexión entre la institución y el resto de la sociedad en algunos ámbitos, aunque con el desarrollo de los Estudios Culturales se ha producido una ampliación en sus campos de estudio incluyendo estas otras formas culturales otrora discriminadas - la literatura popular, por 
ejemplo- . Esto ha posibilitado que la institución —en su concepción tradicional, compuesta de críticos, académicos o revistas especializadasno se haya quedado completamente relegada a un papel secundario en estos procesos culturales, a pesar de que los análisis académicos suelen realizarse en diferido, con un retardo que a veces imposibilita la previsión de los futuros movimientos en el sistema, sobre todo con los nuevos desafíos de la era digital y su inmediatez característica ${ }^{3}$.

La canonización de los productos o modelos culturales se puede producir de acuerdo a dos procedimientos. Por una parte pueden ubicarse directamente en el centro del sistema debido a que comparten ciertas similitudes con los modelos preexistentes. En este caso lo que se produce es una adecuación de las nuevas formas a los modelos previos del sistema. Y, por otro lado, esta llegada puede ser diferida, en el sentido en el que este modelo recién llegado, al no coincidir con los modelos canonizados, se instala en un primer momento en la periferia — donde ejercerá su influjopara luego procederse a su canonización mediante un procedimiento de transferencia. Este segundo caso es de especial interés por ser una clara muestra de dinamismo sistémico. Una obra de arte, si no coincide con los modelos instalados en el centro del sistema, permanecerá en la periferia aguardando el momento para canonizarse. En el instante en el que esto se produzca se dará pues la transferencia, ese modo de proceder tan natural en el polisistema cultural.

Pero la forma más sofisticada de alcanzar ese nivel se da mediante la realización, por productores locales, de productos siguiendo ese modelo importado, lo que provoca que los rasgos foráneos se vayan diluyendo y el modelo vaya adquiriendo matices autóctonos, hasta el punto de que se produce una naturalización en las formas que nos lleva a la noción de interferencia plena. Así, este caso es un ejemplo de las posibles relaciones que se pueden rastrear entre la transferencia y la interferencia durante el proceso de canonización.

En opinión de Sela-Sheffy (2003), para que una interferencia se produzca suelen sucederse tres etapas - aunque no es obligatorio que se den en todos los casos-. La primera se corresponde con la import

\footnotetext{
${ }^{3}$ Así lo vislumbró con acierto Jenaro Talens: "what was once defined in terms of 'serious literature' nowadays has a minor influence in comparison to the electronic discourses that have replaced it as a more attractive and respected source of information" (1995: 5).
} 
of goods or practices, independientemente de la índole que sea, ya sea la importación de un libro, un disco o se produzca la adopción de un extranjerismo léxico. En segundo lugar, se tiene que dar la translation, ya sea interlingüística o de otro tipo, como puede ser la intersemiótica - una adaptación al cine de una novela extranjera, por ejemplo-. Y por último, se debe dar la reproduction, cuando se produce la implementación de un modelo foráneo ya sea en la producción o durante el consumo.

Trasladando esto al ámbito literario, podríamos citar como ejemplo lo ocurrido con el modelo del teatro simbolista en España. Las crónicas de Gómez Carrillo sobre lo que acontecía en el París finisecular provocaron que se despertara un gran interés hacia el éxito del Simbolismo en los escenarios galos. Esto contribuiría a que las obras de Maurice Maeterlinck fueran importadas directamente, editadas en francés, ocupando además un lugar preeminente en las bibliotecas de grandes personalidades culturales de la época ${ }^{4}$. Esta impronta alcanzaría su grado máximo tras la concesión al escritor belga del premio Nobel en 1911. Así, mediante tertulias literarias - una forma de mercado y de consumo en cuanto se pone en relación producto y consumidor - se daría a conocer estas nuevas formas dramáticas, procediéndose a continuación a su traducción al español por autores como Gregorio Martínez Sierra o María de la O Lejárraga, para quienes su influjo es más que evidente en sus creaciones más simbolistas. Si se hace una lectura polisistémica de este caso identificando cada elemento con uno de los factores del sistema, se observa la operatividad de la Teoría de los Polisistemas para desentrañar el funcionamiento de los mecanismos de canonización.

Por otro lado, para que tenga lugar la interferencia tal y como la concebimos, debe producirse una naturalización en su proceso de ejecución (Sela-Sheffy, 2003) . $^{5}$ Desde este punto de vista se dificulta la manipulación del canon, ya que la naturalización del producto implica cierta inconsciencia sobre el origen del modelo si no en su producción, sí al menos en su consumo. Al proponer en este artículo la presencia de

\footnotetext{
${ }^{4}$ Este sería el caso de la biblioteca de la Casa del Arco, donde acudían autoridades literarias como Valle-Inclán a la tertulia de Jesús Muruais, y que albergaba, entre títulos capitales de la literatura francesa, obras importadas de Jarry o Maeterlinck, demostrando su interés en la última dramaturgia internacional (Hormigón, 2006: 91).

${ }^{5}$ Esto conlleva inexorablemente una dificultad extra a la hora de identificar los modelos originarios, ya que los nuevos productos culturales han sido adoptados como propios en el contexto de llegada.
} 
la interferencia como un indicio de canonización, poniendo el énfasis en que su grado de manifestación supremo está vinculado a su acción como guide for cultural production, buscamos una visión más imparcial de este fenómeno. Esto nos obliga a que en nuestro análisis, desde una perspectiva diacrónica, tengamos en cuenta factores como el del productor - que es quién hace uso efectivo del modelo al reconocer su valía artística o comercial — o el mercado — que pone en circulación dicho modelo a través de productos y se los hace llegar al consumidor - que habitualmente son discriminados en los estudios acerca de la canonización.

Para ejemplificar esto y continuando con el caso del Simbolismo en España, los esfuerzos de numerosos escritores por acometer este tipo de teatro (algunos con la demostrada competencia de Lorca o Valle-Inclán) ${ }^{6}$ finalmente fracasan debido a una débil industria teatral incapaz de llevar a buen puerto, y con la solvencia necesaria, la exuberancia formal que este tipo de obras exigía. El mercado se veía pues incapaz de asumir este tipo de productos culturales, provocando que el modelo permaneciera en un high status propio del canon estático, y que los escritores rehusaran acometer este estilo literario ante la dificultad de llevar sus obras a escena y el riesgo de fracaso asociado a este tipo de proyectos.

Volviendo a las ideas de Itamar Even-Zohar, en "Laws of Cultural Interference" (2010), una nueva versión de un artículo publicado originalmente algunas décadas antes (1978), extiende la noción de interferencia polisistémica de la literatura a otros tipos de disciplinas artísticas para toda clase de sociedades. Su objetivo es recopilar algunas leyes generales que están presentes en cualquier proceso de interferencia cultural, y aunque no muestra una excesiva convicción en torno a la posibilidad de descubrir reglas universales, sí en cambio cree en la viabilidad de hallar patrones repetitivos que nos pueden guiar hacia el establecimiento de leyes que nos serían de especial interés. El investigador israelí defiende aquí la existencia de nueve aspectos que nos pueden llevar hacia la definición de Leyes de la Interferencia, que son las que actúan en cualquier encuentro entre sistemas culturales, y que nosotros intentaremos aplicar a nuestro objeto de estudio, la canonización.

En primer lugar, declara que la interferencia siempre es inminente.

${ }^{6}$ Un caso bien conocido es el rotundo fracaso de El maleficio de la mariposa, de Lorca, en 1920, en el Teatro de arte de Gregorio Martínez Sierra y María de la O Lejárraga. 
A veces estas no se muestran de forma evidente, esencialmente porque tienen lugar en la periferia, aunque siempre estarán en el horizonte del sistema completo. El hecho de que la interferencia tenga lugar al margen de los canales no oficiales (no canónicos) de transmisión - como hubiera sido el ámbito académico, por ejemplo - no interrumpe este proceso. El caso de las letras de las canciones de Bob Dylan podría ser muy elocuente a este respecto, pasando de ser uno de los máximos exponentes de la contracultura estadounidense a ser reconocido mundialmente, y de ahí al reconocimiento de la institución al recibir el premio Nobel de Literatura.

De acuerdo a este último aspecto, las interferencias, a su vez, deben actuar en su mayoría de forma unilateral. Si continuamos con el ejemplo del teatro simbolista español, observamos cómo este no influyó en su homólogo francés. Se produce una desconexión y un desinterés desde Francia hacia lo que ocurre en territorio español, pero esto es debido también al rotundo fracaso comercial de los intentos que se llevaron a cabo en España. Aún así, esto no detiene el proceso de canonización, aunque sí hace que se desarrolle por otras vías, y a veces con una falta de sincronía. Un ejemplo de esto lo puede constituir el éxito entre 1945 y 1955 del teatro de Lorca en Francia, siendo uno de los autores más representados de ese momento. Sin embargo, su producción más simbolista no impactaría hasta años más tarde, cuando su obra fuera reconocida como una de las cumbres del teatro universal tras su mitificación como mártir de la guerra civil española (Torres Monreal, 1989), y cuando la deriva de la dramaturgia internacional reclamaba nuevos lenguajes poéticos para sus propuestas escénicas, algo que el teatro lorquiano ya anticipaba, con obras como El público o Comedia sin título.

Atendiendo a esta característica, Even-Zohar declara que no todas las interferencias tienen lugar en todos los niveles de la cultura o, al menos, con la misma intensidad. Esto ocurre especialmente si observamos el funcionamiento de algunos elementos del repertorio dentro de sistemas periféricos como puede ser el correspondiente al colectivo LGTBI (lesbianas, gays, transexuales, bisexuales e intersex). Aquí la noción de ambivalencia de Lotman (1977) se vuelve especialmente operativa. Los modelos que apelan al colectivo LGTBI — temática o incluso formalmente - logran una canonización más rápida y directa dentro de este sistema, pero manteniéndose en la periferia del polisistema completo. Para alcanzar el centro de este debe existir una condición ambivalente 
que permita que dicha obra o modelo pueda ser valorado también por el resto de la población no familiarizada con este tipo de manifestaciones culturales, en igualdad de condiciones y sin que se sientan excluidos, siguiendo la dinámica analizada por Zohar Shavit (1980). Y esto se logra por la presencia de ciertos elementos estructurales que deben coincidir con los de su sistema. En cambio, en los países donde la igualdad de derechos y la naturalización de las diferentes variedades sexuales es una realidad, la interferencia puede tener lugar en cualquier parte del sistema, en cuanto la orientación o condición sexual no es un rasgo excluyente en dicho polisistema. La presencia de personajes homosexuales o transexuales en ficciones del prime time televisivo estadounidense — véase el caso de la serie de Netflix Orange Is the New Black $k^{7}$ - puede ser un significativo ejemplo de este fenómeno ${ }^{8}$.

Even-Zohar expone también cuatro condiciones para que se den las interferencias. De acuerdo a la primera, los contactos generarán interferencias antes o después si no hay circunstancias que provoquen una resistencia firme. Quisiéramos recordar que el concepto de interferencia expuesto por Even-Zohar es más laxo que el propuesto aquí, con lo que si bien es cierto que los contactos crean injerencias de diversa índole, esto no tiene por qué conllevar una canonización de estas formas. Si fuera así, el mundo sería semióticamente ininteligible por la gran cantidad de códigos puestos en acción en cada sistema cultural. A pesar de que estamos defendiendo una organización autónoma del sistema, no podemos entender a este sin la presencia de ciertas estructuras — mutables, eso sí, de ahí su carácter diacrónico - que permiten organizar todo el material significante en una sociedad determinada. En cuanto a las resistencias que se pueden encontrar, estas pueden proceder de diferentes sectores; pueden provenir de los productores, que se niegan a asumir ciertos modelos para sus prácticas artísticas - sería el caso del exilio de escritores tras la instauración de un determinado régimen político contrario a sus ideologías-, o bien del

\footnotetext{
${ }^{7}$ Aún así la canonización entre la población lesbiana ha sido mucho más rápida, al mismo tiempo que contribuye a un cambio en el sistema: "popular media about nontraditional genders are making an awareness of gender diversity more accessible (e.g., the television series Orange is the New Black; Kohan, 2013)" (Levitt e Ippolito, 2014: 1752).

${ }^{8}$ Este comportamiento ambivalente está presente también en el ámbito de la literatura para niños. Tal como analizó Zohar Shavit (1980), esta literatura apela al mismo tiempo a los más jóvenes para quienes se escribe- y a los adultos - los destinatarios reales, ya que determinarán el consumo al ser los responsables tanto de la producción como del mercado o la institución--
} 
mercado, que no ve posibilidades de alcanzar, con los nuevos modelos, ninguno de sus objetivos, ya sean económicos o de otro tipo. La institución también puede oponer una resistencia clara, aunque su fuerza se ve reducida en el mundo globalizado donde el consumidor ${ }^{9}$ ha adquirido una importancia clave al poder dirigir el mercado hacia sus intereses $-\mathrm{y}$ viceversa, el mercado orienta los productos para favorecer la acción del consumidor- ${ }^{10}$.

El quinto aspecto expuesto por Even-Zohar explica que las interferencias son favorecidas cuando un sistema necesita elementos que no están disponibles en su propio repertorio ${ }^{11}$. En España, tras la muerte de Franco, después de años de oscuro nacionalcatolicismo, el arte producido por la subcultura de países como EE. UU. o Reino Unido se convirtió en una gran fuente de recursos para solventar las necesidades de una nueva generación de españoles. Reclamaban nuevos elementos culturales para ser incorporados al repertorio nacional y así ponerse al mismo nivel que el resto del mundo tras años de aislamiento cultural. En esta llegada de

\footnotetext{
${ }^{9}$ Un elemento crucial en la potenciación de la interferencia lo supone una alta disponibilidad del repertorio, algo que está directamente relacionado con el grado de proximidad entre sistemas, lo que contribuye a que el contacto entre ellos sea recurrente y significativo. En la sociedad 3.0 actual, y en su previsible evolución hacia un mundo más interconectado y dinámico aún, resulta difícil hablar de repertorios estancos, ya que su porosidad - excepto en regímenes totalitarios y opacos como puede ser el norcoreano- está en continuo crecimiento, previéndose que alcance proporciones insospechadas si la tendencia actual no se invierte. A través de una simple conexión a internet el repertorio de un joven albanés no dista significativamente mucho del de otro indio. Es el repertorio local el que los diferencia, como el folclore, aunque este no está exento tampoco de las dinámicas sistémicas. Si bien oponen una resistencia mayor por el peso de la institución "tradición", constriñendo su apertura a nuevos modelos, se somete a los mismos mecanismos que rigen el resto del sistema. Por lo tanto hablar de entidades inmutables es contrario a lo que venimos defendiendo aquí. Debemos poner el foco de atención, pues, sobre un repertorio global compartido por toda una generación conectada a través de la red internet, población que está teniendo un papel determinante en los procesos de dinamización cultural.

${ }^{10}$ De nuevo, los ejemplos de gobiernos totalitarios se constituyen en excepciones de esta forma de funcionamiento natural de la interferencia/canonización. El rol de The Little Red Book, de Mao Zedong, en la cultura china durante la dictadura comunista es un ejemplo de cómo la institución puede oponerse al mercado, al productor y al consumidor, en esta ocasión a través de la violencia física e institucional, para imponer sus propios modelos de forma unilateral y sin fisuras.

${ }^{11}$ A este respecto es muy valioso lo dicho por Sela-Sheffy cuando propone que durante el proceso de canonización se pueden incorporar nuevos inventarios que ayuden a la expansión del canon: "In this case, even what appears to be an entirely unprecedented 'innovation' is practically only a 'remodeling' namely, an imposition of existing categories on products that until that point were not labeled as such (while the ultimate effect is eventually still that of enhancing the established rules of the field)" (1994).
} 
repertoremas foráneos (Even-Zohar, 1979) fue determinante que además estos países ocupasen una posición de prestigio $^{12}$. Pero además de por cuestiones de reputación, también dichas interferencias se produjeron por motivos de dominio, como ocurriría por la posición hegemónica de EE.UU. y Reino Unido en todo el concierto internacional desde la Segunda Guerra Mundial hasta nuestros días.

Pero sin lugar a dudas los dos aspectos más interesantes hacen referencia a los procesos y a los procedimientos que actúan en torno a la interferencia. Por una parte la interferencia, que puede tener lugar en una sola área de la cultura de destino, es posible que se extienda a otras. Así, una obra o un conjunto de ellas que crea una interferencia en el sistema literario, puede extenderse y ampliar su radio de acción al resto de sistemas miembros del polisistema. No solo se produce pues una interferencia, sino también una transferencia dentro del polisistema que deviene en una canonización clara. Y por otra parte, un determinado repertorio no tiene por qué mantener necesariamente siempre la misma función que en la cultura original. Trasladado esto a nuestra visión de la interferencia, vemos cómo durante el proceso de importación, traducción y producción naturalizada el producto o modelo originario va sufriendo cambios. Si bien en el primer estadio expuesto por Sela-Sheffy no se modifica el producto, sí se puede producir una modificación en el modo en el que el consumidor recibe ese producto foráneo. Aquí el mercado se encarga de usar sus métodos, como el de la publicidad, para potenciar los rasgos del producto que se adecúan a las expectativas del destinatario final. Con esta mimetización se favorece su asunción y consumo, y por tanto su éxito. Pero es en el segundo estadio cuando se produce una traducción para ajustarlo a las necesidades del sistema targeted, ya que como Gideon Toury dijo, "Obviously, even the most adequacy-oriented translation involves shifts from the source text" (1995: 57). Aunque para Even-Zohar (1990b) estas traducciones siempre tienen una posición periférica, será con la última fase, con la producción naturalizada, cuando se produzca un cambio realmente notable, ya que dicha producción - la interferencia tal y como nosotros la venimos explicando- indicará un cambio de posicionamiento en el sistema, y en

\footnotetext{
${ }^{12}$ Vemos esto también en el ejemplo dado en este artículo acerca del teatro simbolista español, en cuanto lo ocurrido en París tenía un estatus adicional de prestigio y por lo tanto los modelos emanados desde el polisistema francés eran acogidos con una predisposición muy favorable.
} 
este caso su canonización.

\section{EXPRESIONES DE LA INTERFERENCIA}

Analizados diferentes puntos de vista acerca de la interferencia, resulta imprescindible señalar qué formas puede adoptar y qué implicaciones pueden conllevar estas manifestaciones. A la hora de determinar estas interferencias lo más sencillo es bucear en el repertorio cultural para encontrar ejemplos de producción. Recurriendo a la visión de Even-Zohar del repertorio - como un conjunto de reglas y modelos más los productos involucrados en el mismo-, se trataría de identificar aquellos productos culturales que han usado ese modelo para su creación. Estaríamos hablando de la idea de canonicidad dinámica, aquella que se hace presente cuando un modelo u obra es usado como guía en la creación de otras obras de arte. Cuando estos modelos eran originariamente ajenos al sistema donde se crea la obra, se habla, pues de interferencia ${ }^{13}$.

Existen también otras formas intermedias que indican el grado de preeminencia de un texto sobre otros en una cultura determinada. Una de las manifestaciones más llamativas se produce a través del mecanismo de la intermedialidad, un fenómeno que tiene lugar entre la segunda y la tercera etapa del proceso de interferencia descrito por Sela-Sheffy. La intermedialidad entendida como un ejemplo de interferencia se daría cuando un texto, al importarse, es exhibido en una versión mediada y naturalizada. Se trataría en este caso de una traducción intersemiótica en el que el referente original no tiene por qué ser reconocible, aunque otras veces se use como reclamo comercial dado un proceso previo de canonización. Esto es lo que sucedió con las canciones del grupo sueco ABBA, que fueron convertidas en el musical Mamma Mia! Con la dramaturgia de Catherine Johnson, tuvo un exitoso paso por el West End londinense (1999) y el Broadway neoyorquino (2001). Esta recepción tan positiva hizo que fuera adaptada al cine en 2008 bajo el nombre de Mamma Mia! The Movie, dirigida por Phyllida Llyod. El que la empresa fuera llevada a cabo por Universal Studios, hizo que con sus estrategias publicitarias y de distribución las canciones del grupo ABBA vieran reducido su protagonismo, resultando

${ }^{13}$ No se debe confundir la interferencia en este caso con la idea de influencia en Harold Bloom (2009), ya que esta se realiza exclusivamente entre escritores al margen de las dinámicas del sistema. 
para los más jóvenes un producto comercial típico de Hollywood disociado de cualquier vínculo con el célebre grupo sueco. Así paradójicamente, al mismo tiempo que este caso de transmedialidad servía como muestra de la canonización de estas canciones, se potenciaba, por estrategias del mercado, la desaparición de las marcas de sus autores.

Uno de los ejemplos más evidentes de interferencia tiene relación con la presencia en una lengua determinada de neologismos de carácter antropónimo. Nos estamos refiriendo a los casos en los que el nombre del autor es usado para describir un producto o un modelo cultural. Este fenómeno, que no se trata de un extranjerismo al uso, consiste más bien en la incorporación de un autor al vocabulario de otro idioma como consecuencia de la consolidación de su obra en el repertorio del sistema. Piénsese en el ámbito fílmico con adjetivos en inglés como Hitchcknonian o Almodóvarian, o bien en el teatro con Brechtian. Lo mismo sucede si pensamos en las artes plásticas. Todos se constituyen en muestra de cómo una obra o autor puede alcanzar un estatus tal dentro de un sistema cultural determinado hasta el punto de que su propio nombre, mediante el neologismo, se asocia al modelo que ha generado.

Pero lo que más nos interesa de este fenómeno es cuando ese procedimiento se produce además en contextos alejados del ámbito en el que surge. Es decir, cuando ya no se usa en un sector especializado, sino que su aplicación se realiza en otros entornos más amplios. Así, es más que posible que se extienda el uso de este tipo de adjetivos a otros entornos y en contextos diversos, lo que indica que se ha producido una semantización clara del adjetivo, no requiriéndose el conocimiento del referente original para entender lo que significa. Podríamos constatar este comportamiento en el amplio uso de Kafkaesque, refiriéndose a una situación irracional y angustiosa, o en el ámbito francés con el adjetivo Ubuesque, en referencia al célebre personaje de Alfred Jarry y para aludir a situaciones totalmente absurdas.

Esta forma supondría una expresión clara de lo que es la canonización en un grado superlativo, en cuanto no actúa ninguna fuerza rectora de forma directa, al modo del canon clásico, sino que son las dinámicas sistémicas las que determinan el uso de tal o cual elemento del repertorio. El lenguaje es una entidad que aún en contextos no democráticos adquiere un comportamiento autónomo, a pesar de las presiones ejercidas desde el poder — político o académico- para controlar las dinámicas de su génesis 
y uso, de tal forma que, salvo en algunos contextos, el lenguaje refleja la vida y sus dinámicas, y no a la inversa. El que se constate esta serie de neologismos es indicativo de que en el polisistema se ha producido un cambio, identificado con una interferencia que nos demuestra la canonización de una obra o un modelo y que nos aproxima a la posibilidad de un canon epistémico como el enunciado por Walter Mignolo (1991).

\section{REFERENCIAS BIBLIOGRÁFICAS}

BLOOM, H. (1994). The Western Canon: The Books and School of the Ages. New York-San Diego-London: Harcourt Brace \& Company. (2009). La ansiedad de la influencia: una teoría de la poesía. Madrid: Trotta.

EVEN-ZOHAR, I. (1978). "Laws of Literary Interference". Poetics Today 11.1, 53-72.

(1979). "Textemic Status of Signs in a Literary Text and its Translation". A Semiotic Landscape: Proceedings of the First Congress of the IASS, S. Chatman, U. Eco y J. M. Klinkenberg (eds.), 629-633. The Hague: Mouton.

(1990a). "Polysystem Theory". Poetics Today 11.1, 9-26.

(1990b). "The Position of Translated Literature within the Literary Polysystem". Poetics Today 11.1, 45-51.

(1997). "Factors and Dependencies in Culture: A Revised Outline for Polysystem Culture Research". Canadian Review of Comparative Literature/Revue Canadienne de Littérature Comparée 24.1, 1534.

(2010). "Laws of cultural interference". En Papers in Culture Research, I. Even-Zohar, 52-69. Tel Aviv: Unit of Culture ResearchTel Aviv University.

HORMIGÓN, J. A. (2006). Valle-Inclán: Biografia cronológica y Epistolario I (1866-1919). Madrid: Asociación de Directores de Escena de España.

JAKOBSON, R. (1960). "Closing Statement: Linguistics and Poetics". En Style in Language, T. A. Sebeok (ed.), 350-377. Cambridge: M.I.T. JAUSS, H. R. (2013). La historia de la literatura como provocación. Madrid: Gredos. 
KERMODE, F. (1998). "El control institucional de la interpretación". En El canon literario, Sullà, E. (comp.), 91-112. Madrid: Arco / Libros. LEVITT, H. M. e IPPOLITO, M. R. (2014). "Being Transgender: The Experience of Transgender Identity Development". Journal of Homosexuality 61, 1727-1758.

LOTMAN, J. (1977). "The Dynamic Model of a Semiotic System". Semiotica 21, 3.4, 193-210.

MIGNOLO, W. (1991). "Canons a(nd) cross-cultural boundaries (or, whose canon are we talking about?)". Poetics Today 12.1, 1-28.

POZUELO YVANCOS, J. M. (1995). El canon en la teoría literaria contemporánea. Valencia: Episteme.

(2000). "El canon en las teorías sistémicas". En Teoría del canon y literatura española, J. M. Pozuelo Yvancos y R.M. Aradra Sánchez (eds.), 77-90. Madrid: Cátedra.

(2006). "Canon e historiografía literaria". Mil Seiscientos Dieciséis 11, 17-28.

SELA-SHEFFY, R. (1994). "Strategies of Canonization: manipulating the idea of the novel and the intellectual field in eighteen century German culture". http://www.tau.ac.il/ rakefet/papers/rs-strat.htm [10/01/2020].

(2002). "Canon formation revisited: canon and cultural production". Neohelicon 29.2, 141-159.

(2003). "Interference and Aspects of Repertoire Consolidation in Culture". The Challenge of the Hyksos: cultural interference in the New Kingdom, B. Manfred y O. Goldwasser (eds.), s. p. Vienna: Austrian Academy of Sciences. www.tau.ac.il/ rakefet/papers/RSInterference.pdf [10/01/2020].

SHAVIT, Z. (1980). "The Ambivalent Status of Texts: The Case of Children's Literature". Poetics Today 1.3, 75-86.

SULLÁ, E. (1998). “El debate sobre canon literario". En El canon literario, E. Sullá (comp.), 11-34. Madrid: Arco / Libros.

TALENS, J. (1995). "Writing against Simulacrum: The place of literature and literary theory in the Electronic Age". Boundary 2.1, 1-21.

TOURY, G. (1995). "The Nature and Role of Norms in Translation". En Descriptive Translation Studies and Beyond, G. Toury, 53-69. Amsterdam: Philadelphia: John Bejamins.

TORRES MONREAL, F. (1989). "El teatro de García Lorca en Francia 
(1938-1973)”. Estudios románicos 5, 1346-1369.

TÖTÖSY DE ZEPETNEK, S. (1992). "Systemic Approaches to Literature. An Introduction with Selected Bibliographies". Canadian Review of Comparative Literature/Revue Canadienne de Littérature Comparée 19, 1-2, 21-93.

Recibido el 18 de enero de 2020.

Aceptado el 20 de abril de 2020. 